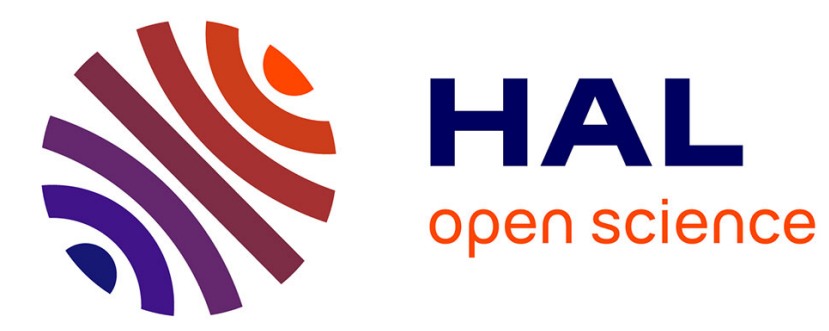

\title{
Immersion and Invariance Observers for Gyro-Free Attitude Control Systems
}

\author{
Sungpil R Yang, Maruthi R Akella, Frédéric R Mazenc
}

\section{To cite this version:}

Sungpil R Yang, Maruthi R Akella, Frédéric R Mazenc. Immersion and Invariance Observers for Gyro-Free Attitude Control Systems. Journal of Guidance, Control, and Dynamics, 2016, 39, pp.2570 - 2577. 10.2514/1.G002095 . hal-01389858

\section{HAL Id: hal-01389858 https://hal.inria.fr/hal-01389858}

Submitted on 30 Oct 2016

HAL is a multi-disciplinary open access archive for the deposit and dissemination of scientific research documents, whether they are published or not. The documents may come from teaching and research institutions in France or abroad, or from public or private research centers.
L'archive ouverte pluridisciplinaire HAL, est destinée au dépôt et à la diffusion de documents scientifiques de niveau recherche, publiés ou non, émanant des établissements d'enseignement et de recherche français ou étrangers, des laboratoires publics ou privés. 


\title{
Immersion and Invariance Observers for Gyro-Free Attitude Control Systems
}

\author{
Sungpil Yang* and Maruthi R. Akella ${ }^{\dagger}$ \\ University of Texas at Austin, Austin, Texas, 78712 \\ Frédéric Mazenc ${ }^{\ddagger}$ \\ EPI DISCO INRIA-Saclay, 91192, Gif-sur-Yvette, France
}

\section{Introduction}

The rigid-body attitude control problem has been extensively studied due to mathematical interest in the study of kinematics, geometric mechanisms, and a wide range of challenging applications such as spacecraft, underwater vehicle and robot manipulator systems [1,2]. Although this problem is now considered to be well understood, it still attracts researchers as control theory develops and applications are diversified. The theoretical difficulties associated with this problem originate not only from the nonlinearity of the dynamics itself, but also from challenging practical constraints additionally imposed to achieve certain objectives - e.g., partial availability of states, system uncertainty, degradation or saturation of actuators [3-9].

In the case where angular velocity measurements are not readily available for control implementation, numerous output feedback controllers have been proposed only utilizing attitude measurements. Recently, it was shown that a set of unit vector measurements can be directly used in a feedback control law without constructing an attitude vector to regulate the body orientation $[10,11]$. Such "gyro-free" controllers are mainly based on construction of auxiliary filters or dynamic observers. Thanks to certain passivity properties of the dynamics, output of an attitude

${ }^{*} \mathrm{Ph} . \mathrm{D}$. Candidate, Department of Aerospace Engineering and Engineering Mechanics, 1 University Station; yang.sungpil@utexas.edu. Student Member AIAA

†Professor, Department of Aerospace Engineering and Engineering Mechanics, 1 University Station. Myron L. Begeman Fellow in Engineering; makella@mail.utexas.edu. Associate Fellow AIAA

${ }^{\sharp}$ Charge de Resherche, The Laboratoire des Signaux et Systèmes (L2S, UMR CNRS 8506), CNRS, CentraleSupélec, Université Paris-Sud, 3 rue Joliot Curie; frederic.mazenc@12s.centralesupelec.fr; Mazenc was supported by l'Institut pour le Contrôle et la Décision de l'Idex Paris-Saclay (iCODE). 
error filter can be used as a replacement for the rate error feedback [3-5]. Alternatively, angular velocity can be estimated regardless of control input through an observer and the estimates are treated in certainty-equivalence fashion as true rate measurements $[12,13]$. Compared to passivity-based controllers, the observer-controller combination requires establishment of a separation property which is not automatically guaranteed for nonlinear systems.

Recently in [14], an exponentially converging angular velocity observer was proposed based on Immersion and Invariance (I\&I) design aided by a new dynamic scaling method suggested in [15]. Readers are referred to $[16,17]$ for further details of the general I\&I methodology and to [18, 19] for nonlinear observer applications. Using switching logic, a $C^{0}$ continuous angular velocity observer with an exponential convergence was designed in [20] while a $C^{\infty}$ observer was suggested showing an asymptotic convergence of the estimation errors in [21]. The aforementioned observers are driven by quaternion measurements and combined with proportional-derivative (PD) type controllers establishing an almost global asymptotic stability for the overall closed-loop system.

In this paper, a new lower dimensional I\&I observer is proposed based on a novel definition of the angular velocity estimate. Instead of using a filter for the angular velocity estimate to dominate Coriolis effect suggested in [14], the direction cosine matrix associated with the quaternion state is utilized so that cross terms caused by both Coriolis effect and an approximate solution to a partial differential equation (PDE) I\&I design inevitably involves are expressed in terms of quaternion filter error in Lyapunov analysis. Consequently, the observer dynamics evolve only in 8-dimensional space instead of the 11-dimensional space in [14] and stability analysis becomes simpler due to the fact that the dynamics of the scaling factor are only driven by the quaternion filter error. Moreover, further simplification is made by utilizing a structural property of the quaternion kinematics. Typically, I\&I design with dynamic scaling requires additional term in the time-varying gain associated with the quaternion filter in order to bound the scaling factor [15], but the added term is shown to be zero for this particular application. When this observer is equipped with a PD tracking control, a separation property is established through the use of a partially strictified Lyapunov function candidate which is similar to one in [22].

This note is organized as follows. The rigid-body rotational dynamics and the corresponding 
quaternion kinematics are described in Section II and some useful lemmas are presented in Section III. Then the I\&I observer design is discussed in Section IV. The stability analysis of the PD feedback control is conducted both with true rate measurements in Section V and with its estimates in Section VI, which also establishing the separation property. Section VII provides numerical results showing the performance of the proposed observer and controller. Finally, we ends with some concluding remarks in Section VIII.

\section{Model Description}

Consider the rigid-body rotational motion. Let $\omega(t) \in \mathbb{R}^{3}$ and $\mathbf{u}(t) \in \mathbb{R}^{3}$ be the angular rate and the applied control torque respectively both represented in the body-fixed frame. Then the dynamics are governed by Euler's rotational equations of motion given by

$$
J \dot{\omega}(t)=-S(\omega(t)) J \omega(t)+\mathbf{u}(t)
$$

where $J \in \mathbb{R}^{3 \times 3}$ is the positive definite inertia matrix and $S: \mathbb{R}^{3} \rightarrow \mathbb{R}^{3 \times 3}$ is the skew-symmetric matrix operator such that it is equivalent to the vector cross product, i.e., $S(\mathbf{x}) \mathbf{y}=\mathbf{x} \times \mathbf{y}$ for any $\mathbf{x}, \mathbf{y} \in \mathbb{R}^{3}$.

In this note, the quaternion kinematics are adopted to avoid the singularity:

$$
\dot{\mathbf{q}}(t)=\frac{1}{2} E(\mathbf{q}(t)) \omega(t)
$$

where the quaternion $\mathbf{q}(t) \in \mathbb{Q}=\left\{\mathbf{q}=\left(q_{0}, \mathbf{q}_{v}\right) \in \mathbb{R} \times \mathbb{R}^{3} \mid \mathbf{q}^{T} \mathbf{q}=1\right\}$ represents the orientation of the body frame $\mathcal{F}_{B}$ with respect to the inertial frame $\mathcal{F}_{I}$. The matrix function $E: \mathbb{R} \times \mathbb{R}^{3} \rightarrow \mathbb{R}^{4 \times 3}$ in Eq. (2) is defined by

$$
E(\mathbf{q})=\left[\begin{array}{c}
-\mathbf{q}_{v}^{T} \\
S\left(\mathbf{q}_{v}\right)+q_{0} I_{3}
\end{array}\right],
$$

where $I_{3}$ is the 3-dimensional identity matrix. Note that, given a quaternion $\mathbf{q} \in \mathbb{Q}$, a direction 
cosine matrix which also represents the body attitude can be obtained by the following identity:

$$
C(\mathbf{q})=I_{3}-2 q_{0} S\left(\mathbf{q}_{v}\right)+2 S^{2}\left(\mathbf{q}_{v}\right)
$$

whose corresponding kinematics are given by

$$
\dot{C}(\mathbf{q}(t))=-S(\omega(t)) C(\mathbf{q}(t))
$$

In our formulation, it is assumed that the inertia matrix $J$ is exactly known and only quaternion measurements are available from attitude sensors. The objective is to design an observer that estimates the states $\mathbf{q}(t)$ and $\omega(t)$ with an exponential convergence using the I\&I design method.

From the subsequent section on, function arguments are often dropped for notational simplicity.

\section{Preliminaries}

Before presenting our main result, we state some important technical results to make our analysis easier to follow. Detailed proofs are omitted in the interest of brevity.

Lemma 1. Let $S O(3)$ be the 3-dimensional rotation group defined as

$$
S O(3)=\left\{R \in \mathbb{R}^{3 \times 3} \mid R^{T} R=I_{3}, \operatorname{det}\{R\}=1\right\} .
$$

For any $R \in S O(3)$ and $\mathbf{x} \in \mathbb{R}^{3}$

$$
R S(\mathbf{x})=S(R \mathbf{x}) R
$$

In other words, the vector cross product in three-dimension is invariant under proper rotations.

Lemma 2. For any symmetric matrix $M \in \mathbb{R}^{3 \times 3}$ and any vector $\mathbf{x} \in \mathbb{R}^{3}$, the equality

$$
S(M \mathbf{x})+M S(\mathbf{x})+S(\mathbf{x}) M=\operatorname{Tr}(M) S(\mathbf{x})
$$

holds, where $\operatorname{Tr}(M)$ is the trace of the matrix $M$. 
Lemma 3. Consider the function $E(\mathbf{q})$ defined in Eq. (3). For any $\mathbf{q} \in \mathbb{R}^{4}$, the following equalities are satisfied:

$$
\begin{aligned}
E^{T}(\mathbf{q}) \mathbf{q} & =\mathbf{0}, \\
\|E(\mathbf{q})\|_{2} & =\|\mathbf{q}\|_{2} .
\end{aligned}
$$

Note Eq. (9) indicates that the unit-norm constraint is invariant under the quaternion kinematics. It is induced by Eqs. (3) and (10) that the function $E(\mathbf{q})$ is globally Lipschitz in $\mathbf{q}$.

\section{I\&I Observer Design}

\section{A. Observer states}

Let $\hat{\mathbf{q}}(t)$ and $\hat{\boldsymbol{\omega}}(t)$ be the observer states that track $\mathbf{q}(t)$ and $\omega(t)$ respectively. Then the errors are defined as

$$
\begin{gathered}
\tilde{\mathbf{q}}=\hat{\mathbf{q}}-\mathbf{q}, \\
\tilde{\boldsymbol{\omega}}=\hat{\omega}-\omega .
\end{gathered}
$$

The angular velocity estimate is assumed to be

$$
\hat{\omega}=C(\mathbf{q}) \bar{\omega}+\beta(\hat{\mathbf{q}}, \mathbf{q})
$$

where the mapping $\boldsymbol{\beta}: \mathbb{R}^{4} \times \mathbb{Q} \rightarrow \mathbb{R}^{3}$ is to be determined. The objective is to design $\dot{\hat{\mathbf{q}}}(t), \dot{\overline{\boldsymbol{\omega}}}(t)$ and $\beta(t)$ such that

$$
\lim _{t \rightarrow \infty}(\tilde{\mathbf{q}}(t), \tilde{\boldsymbol{\omega}}(t))=(\mathbf{0}, \mathbf{0})
$$




\section{B. Observer error dynamics}

Let us consider the time-derivative of the angular velocity estimate defined in Eq. (13). Using Eq. (5), we obtain

$$
\begin{aligned}
\dot{\hat{\omega}} & =\dot{C}(\mathbf{q}) \overline{\boldsymbol{\omega}}+C(\mathbf{q}) \dot{\bar{\omega}}+\dot{\boldsymbol{\beta}}(\hat{\mathbf{q}}, \mathbf{q}) \\
& =-S(\omega) C(\mathbf{q}) \overline{\boldsymbol{\omega}}+C(\mathbf{q}) \dot{\bar{\omega}}+\dot{\boldsymbol{\beta}} .
\end{aligned}
$$

After arranging terms in the above equation, we have

$$
\dot{\hat{\omega}}=S(\tilde{\boldsymbol{\omega}})(\hat{\boldsymbol{\omega}}-\boldsymbol{\beta})-S(\hat{\boldsymbol{\omega}}) C(\mathbf{q}) \overline{\boldsymbol{\omega}}+C(\mathbf{q}) \dot{\bar{\omega}}+\dot{\boldsymbol{\beta}}
$$

which leads to

$$
\begin{aligned}
J \dot{\tilde{\omega}} & =J \dot{\hat{\omega}}-J \dot{\omega} \\
& =J S(\tilde{\omega})(\hat{\boldsymbol{\omega}}-\boldsymbol{\beta})-J S(\hat{\boldsymbol{\omega}}) C(\mathbf{q}) \bar{\omega}+J C(\mathbf{q}) \dot{\bar{\omega}}+J \dot{\beta}+S(\hat{\omega}-\tilde{\omega}) J(\hat{\boldsymbol{\omega}}-\tilde{\boldsymbol{\omega}})-\mathbf{u}
\end{aligned}
$$

Once Lemma 2 is applied and the following relationships are substituted:

$$
\begin{aligned}
& \bar{J}=\operatorname{Tr}(J) I_{3}-2 J \\
& \boldsymbol{\mu}=C(\mathbf{q}) \dot{\bar{\omega}}-S(\hat{\boldsymbol{\omega}}) C(\mathbf{q}) \overline{\boldsymbol{\omega}}+J^{-1}(S(\hat{\boldsymbol{\omega}}) J \hat{\boldsymbol{\omega}}-\mathbf{u})
\end{aligned}
$$

the rate error dynamics are obtained as

$$
J \dot{\tilde{\omega}}=S(\tilde{\boldsymbol{\omega}})(J \tilde{\boldsymbol{\omega}}+\bar{J} \hat{\boldsymbol{\omega}})-J S(\tilde{\boldsymbol{\omega}}) \boldsymbol{\beta}+J(\boldsymbol{\mu}+\dot{\boldsymbol{\beta}})
$$

We will design $\boldsymbol{\mu}(t)$ and then recover $\dot{\overline{\boldsymbol{\omega}}}(t)$ through the following relation:

$$
\dot{\bar{\omega}}=C^{T}(\mathbf{q})\left[\boldsymbol{\mu}+S(\hat{\boldsymbol{\omega}}) C(\mathbf{q}) \overline{\boldsymbol{\omega}}-J^{-1}(S(\hat{\boldsymbol{\omega}}) J \hat{\boldsymbol{\omega}}-\mathbf{u})\right]
$$


Remark 1. The key idea of this work compared to the result in [14] is that $\boldsymbol{\beta}$ is a function of only $\hat{\mathbf{q}}$ and $\mathbf{q}$, so the observer does not require a three-dimensional filter for $\hat{\boldsymbol{\omega}}$ such that the filter state converges to $\hat{\omega}$ as time goes to infinity. This is achieved by use of the direction cosine matrix associated with the quaternion state to define the rate estimate in Eq. (13). We prove in the following analysis that the second term $J S(\tilde{\omega}) \boldsymbol{\beta}$ in the right-hand side of Eq. (20) will be managed by designing $\boldsymbol{\beta}$ such that $\boldsymbol{\beta} \rightarrow \mathbf{0}$ as $\tilde{\mathbf{q}} \rightarrow \mathbf{0}$ without introducing extra dynamic extension.

We also propose the dynamics for the quaternion filter as

$$
\dot{\hat{\mathbf{q}}}(t)=-k_{q} r^{2}(t) \tilde{\mathbf{q}}(t)+\frac{1}{2} E(\mathbf{q}(t)) \hat{\boldsymbol{\omega}}(t), \quad \hat{\mathbf{q}}(0)=\mathbf{q}(0)
$$

where $k_{q}$ is a positive constant and $r(t)$ is the dynamic scaling factor to be determined in the following section. Then the quaternion error dynamics become

$$
\dot{\tilde{\mathbf{q}}}=-k_{q} r^{2} \tilde{\mathbf{q}}+\frac{1}{2} E(\mathbf{q}) \tilde{\boldsymbol{\omega}}
$$

\section{Dynamic scaling}

The original I\&I methodology requires the existence of a function $\boldsymbol{\beta}(\mathbf{q})$ such that

$$
\frac{\partial \boldsymbol{\beta}}{\partial \mathbf{q}}=k_{\omega} E^{T}(\mathbf{q})
$$

where $k_{\omega}$ is a positive constant. However, no such solution to the above PDE exists. In order to apply I\&I methodology, we rather consider an approximate solution $\boldsymbol{\beta}(\hat{\mathbf{q}}, \mathbf{q})$ such that

$$
\frac{\partial \boldsymbol{\beta}}{\partial \mathbf{q}}=k_{\omega} E^{T}(\hat{\mathbf{q}})
$$

and $\lim _{t \rightarrow \infty} \hat{\mathbf{q}}(t)=\mathbf{q}(t)$. The effect caused by this approximation will be managed by introducing a dynamic scaling factor to be discussed later.

Let

$$
\boldsymbol{\beta}=k_{\omega} E^{T}(\hat{\mathbf{q}}) \mathbf{q}
$$


and

$$
\boldsymbol{\mu}=k_{\omega} E^{T}(\tilde{\mathbf{q}})\left(k_{q} r^{2} \mathbf{q}-\frac{1}{2} E(\mathbf{q}) \hat{\boldsymbol{\omega}}\right) .
$$

Since

$$
\begin{aligned}
\dot{\boldsymbol{\beta}} & =k_{\omega} E^{T}(\hat{\mathbf{q}}) \dot{\mathbf{q}}-k_{\omega} E^{T}(\mathbf{q}) \dot{\hat{\mathbf{q}}} \\
& =-\frac{k_{\omega}}{2} E^{T}(\hat{\mathbf{q}}) E(\mathbf{q}) \tilde{\boldsymbol{\omega}}-k_{\omega} E^{T}(\tilde{\mathbf{q}})\left(k_{q} r^{2} \mathbf{q}-\frac{1}{2} E(\mathbf{q}) \hat{\boldsymbol{\omega}}\right) \\
& =-\frac{k_{\omega}}{2} \tilde{\boldsymbol{\omega}}-\frac{k_{\omega}}{2} E^{T}(\tilde{\mathbf{q}}) E(\mathbf{q}) \tilde{\boldsymbol{\omega}}-k_{\omega} E^{T}(\tilde{\mathbf{q}})\left(k_{q} r^{2} \mathbf{q}-\frac{1}{2} E(\mathbf{q}) \hat{\boldsymbol{\omega}}\right),
\end{aligned}
$$

the closed-loop dynamics are obtained as

$$
J \dot{\tilde{\omega}}=S(\tilde{\boldsymbol{\omega}})(J \tilde{\boldsymbol{\omega}}+\bar{J} \hat{\boldsymbol{\omega}})-k_{\omega} J S(\tilde{\boldsymbol{\omega}}) E^{T}(\hat{\mathbf{q}}) \mathbf{q}-\frac{k_{\omega}}{2} J \tilde{\boldsymbol{\omega}}-\frac{k_{\omega}}{2} J E^{T}(\tilde{\mathbf{q}}) E(\mathbf{q}) \tilde{\boldsymbol{\omega}}
$$

Now consider $V_{\tilde{\omega}}=(1 / 2) \tilde{\boldsymbol{\omega}} J \tilde{\boldsymbol{\omega}}$ whose time-derivative is given by

$$
\dot{V}_{\tilde{\boldsymbol{\omega}}}=-\frac{k_{\omega}}{2}\left\|J^{1 / 2} \tilde{\boldsymbol{\omega}}\right\|_{2}^{2}-k_{\omega} \tilde{\boldsymbol{\omega}}^{T} J S(\tilde{\boldsymbol{\omega}}) E^{T}(\hat{\mathbf{q}}) \mathbf{q}-\frac{k_{\omega}}{2} \tilde{\boldsymbol{\omega}}^{T} J E^{T}(\tilde{\mathbf{q}}) E(\mathbf{q}) \tilde{\boldsymbol{\omega}}
$$

Since $V_{\tilde{\omega}}$ is not upper bounded by a non-positive function, it cannot be further analyzed. In oder to handle the sign indefinite terms in Eq. (30) we introduce, using the dynamic scaling factor $r(t)$, the scaled rate error defined as

$$
\mathbf{z}(t)=\frac{1}{r(t)} \tilde{\boldsymbol{\omega}}(t)
$$

and propose

$$
\dot{r}=-k_{1} k_{\omega}(r-1)+k_{2} k_{\omega}\|\tilde{\mathbf{q}}\|_{2}^{2} r, \quad r(0)=1
$$

where $k_{1}$ and $k_{2}$ are positive constants. Note that, since the set $\{r \in \mathbb{R} \mid r \geq 1\}$ is positively invariant, the choice of the initial condition ensures $r(t) \geq 0$ for all $t \geq 0$. Along the trajectories of Eqs. (29) and (32), we have

$$
J \dot{\mathbf{z}}=S(\mathbf{z})(J \tilde{\boldsymbol{\omega}}+\bar{J} \hat{\boldsymbol{\omega}})-\frac{k_{\omega}}{2} J \mathbf{z}-\frac{\dot{r}}{r} J \mathbf{z}-k_{\omega} J S(\mathbf{z}) E^{T}(\hat{\mathbf{q}}) \mathbf{q}-\frac{k_{\omega}}{2} J E^{T}(\tilde{\mathbf{q}}) E(\mathbf{q}) \mathbf{z}
$$


Then the time-derivative of the Lyapunov-like function defined by

$$
V_{\mathbf{z}}=\frac{1}{2 k_{\omega}} \mathbf{z}^{T} J \mathbf{z}
$$

is obtained as

$$
\dot{V}_{\mathbf{z}}=-\left(\frac{1}{2}-\frac{k_{1}(r-1)}{r}\right) \mathbf{z}^{T} J \mathbf{z}-k_{2}\|\tilde{\mathbf{q}}\|_{2}^{2} \mathbf{z}^{T} J \mathbf{z}-\mathbf{z}^{T} J S(\mathbf{z}) E^{T}(\hat{\mathbf{q}}) \mathbf{q}-\frac{1}{2} \mathbf{z}^{T} J E^{T}(\tilde{\mathbf{q}}) E(\mathbf{q}) \mathbf{z}
$$

Let $J_{M}$ and $J_{m}$ denote the largest and the smallest eigenvalues of the inertia matrix $J$ so that the inequalities

$$
J_{m}\|\mathbf{z}\|_{2}^{2} \leq \mathbf{z}^{T} J \mathbf{z} \leq J_{M}\|\mathbf{z}\|_{2}^{2}
$$

and

$$
\|S(\mathbf{z}) J \mathbf{z}\|_{2}^{2} \leq J_{\Delta}\|\mathbf{z}\|_{2}^{2} \mathbf{z}^{T} J \mathbf{z}
$$

hold for any $\mathbf{z} \in \mathbb{R}^{3}$, where $J_{\Delta}=J_{M}-J_{m}$. Using the above inequalities and the fact that

$$
\begin{aligned}
\left\|E^{T}(\hat{\mathbf{q}}) \mathbf{q}\right\|_{2} & =\left\|E^{T}(\mathbf{q}) \mathbf{q}+E^{T}(\tilde{\mathbf{q}}) \mathbf{q}\right\|_{2} \\
& \leq\|\tilde{\mathbf{q}}\|_{2}
\end{aligned}
$$

from Lemma 3, the upper bound of $V_{\mathbf{z}}$ is obtained as

$$
\dot{V}_{\mathbf{z}} \leq-\left[\begin{array}{c}
\mathbf{z} \\
\|\tilde{\mathbf{q}}\| \mathbf{z}
\end{array}\right]^{T}\left(Q_{1} \otimes J\right)\left[\begin{array}{c}
\mathbf{z} \\
\|\tilde{\mathbf{q}}\| \mathbf{z}
\end{array}\right]
$$

where

$$
Q_{1}=\left[\begin{array}{cc}
\frac{1}{2}-k_{1} & -\frac{J_{M}+2 \sqrt{J_{m} J_{\Delta}}}{4 J_{m}} \\
-\frac{J_{M}+2 \sqrt{J_{m} J_{\Delta}}}{4 J_{m}} & k_{2}
\end{array}\right]
$$

and the operator $\otimes$ denotes the Kronecker product.

Remark 2. As mentioned in Remark 1, the choice of $\boldsymbol{\beta}$ in Eq. (26) renders the cross term caused by the Coriolis effect to depend on $\tilde{\mathbf{q}}$ and $\mathbf{z}$. Similarly, the other cross term coming from the 
time derivative of the approximate solution $\boldsymbol{\beta}(\hat{\mathbf{q}}, \mathbf{q})$ is a function of $\tilde{\mathbf{q}}$ and $\mathbf{z}$. Together they give r-dynamics driven by $\|\tilde{\mathbf{q}}\|_{2}^{2}$ only.

If $k_{1}$ and $k_{2}$ are additionally constrained as

$$
\begin{aligned}
& k_{1}<\frac{1}{2}, \\
& k_{2}>\frac{\left(J_{M}+2 \sqrt{J_{m} J_{\Delta}}\right)^{2}}{8 J_{m}^{2}\left(1-2 k_{1}\right)},
\end{aligned}
$$

the matrix $Q_{1}$ becomes positive definite, and thus there exists a finite constant $c_{1}>0$ such that

$$
\dot{V}_{\mathbf{z}} \leq-c_{1}\|\mathbf{z}\|_{2}^{2}
$$

Moreover, since

$$
\dot{V}_{\mathbf{z}} \leq-\frac{2 k_{\omega} c_{1}}{J_{M}} V_{\mathbf{z}}
$$

the norm of $\mathbf{z}(t)$ is bounded by

$$
\|\mathbf{z}(t)\|_{2} \leq\|\mathbf{z}(0)\|_{2} \sqrt{\frac{J_{M}}{J_{m}}} \exp \left\{-\frac{k_{\omega} c_{1}}{J_{M}} t\right\},
$$

which implies that $\mathbf{z}(t)$ converges to zero exponentially fast.

To show the convergence of $\tilde{\boldsymbol{\omega}}$, consider

$$
V_{o}=\frac{1}{2} \tilde{\mathbf{q}}^{T} \tilde{\mathbf{q}}+\zeta_{1} V_{\mathbf{z}}
$$

where $\zeta_{1}>1 /\left(8 k c_{1}\right)$. Along the trajectories of the closed-loop dynamics the time-derivative of $V_{o}$ yields

$$
\dot{V}_{o}=-k_{q} r^{2}\|\tilde{\mathbf{q}}\|_{2}^{2}+\frac{r}{2} \tilde{\mathbf{q}}^{T} E(\mathbf{q}) \mathbf{z}+\zeta_{1} \dot{V}_{\mathbf{z}}
$$


This is upper bounded by

$$
\dot{V}_{o} \leq-\frac{k_{q}}{2} r^{2}\|\tilde{\mathbf{q}}\|_{2}^{2}-\left[\begin{array}{c}
r\|\tilde{\mathbf{q}}\|_{2} \\
\|\mathbf{z}\|_{2}
\end{array}\right]^{T} Q_{2}\left[\begin{array}{c}
r\|\tilde{\mathbf{q}}\|_{2} \\
\|\mathbf{z}\|_{2}
\end{array}\right] \text {, }
$$

where

$$
Q_{2}=\left[\begin{array}{cc}
\frac{k}{2} & -\frac{1}{4} \\
-\frac{1}{4} & \zeta_{1} c_{1}
\end{array}\right]
$$

is positive definite.

\section{Boundedness of scaling factor}

It is essential to establish the boundedness of $r(t)$ to show the convergence of $\tilde{\mathbf{q}}(t)$ since Eqs. (46) and (48) are only valid as long as $r(t)$ is finite. To prove $r \in L_{\infty}$, we construct another Lyapunov function candidate

$$
W=V_{o}+\frac{k_{q}}{8 k_{2} k_{\omega}} r^{2}
$$

whose time derivative is given by

$$
\begin{aligned}
\dot{W} & =\dot{V}_{o}-\frac{k_{1} k_{q}}{4 k_{2} k_{\omega}} r(r-1)+\frac{k_{q}}{4} r^{2}\|\tilde{\mathbf{q}}\|_{2}^{2} \\
& \leq-\frac{k_{q}}{4} r^{2}\|\tilde{\mathbf{q}}\|_{2}^{2} .
\end{aligned}
$$

Since $W(t)$ is non-increasing, the scaling factor is bounded for all $t \in[0, \infty)$.

Remark 3. To ensure $r \in L_{\infty}$, the quaternion estimate dynamics typically have the form

$$
\dot{\hat{\mathbf{q}}}(t)=-K(t) \tilde{\mathbf{q}}(t)+\frac{1}{2} E(\mathbf{q}(t)) \hat{\boldsymbol{\omega}}(t)
$$

with

$$
K(t)=k_{q} r^{2}(t)+\epsilon r^{2}(t) \psi(\mathbf{q}(t), \tilde{\mathbf{q}}(t))
$$

where $\epsilon$ is a positive constant and $\psi$ is a function that cancels the positive term in $\dot{W}$ [15]. However, 
owing to the fact that the function $E(\mathbf{q})$ is globally Lipschitz, the increasing term in the $\dot{r}$ equation becomes proportional to $\|\tilde{\mathbf{q}}\|_{2}^{2}$ instead of $\|E(\tilde{\mathbf{q}})\|_{2}^{2}$, and thus the additional term $\psi$ can be set to be zero so that the first term in the dynamic gain $K(t)$ absorbs the other.

\section{E. Convergence properties}

As $r(t)$ is finite for all $t \geq 0$, standard Lyapunov analysis is now applied. The positive definite matrix $Q_{2}$ in Eq. (48) allows us to find some $c_{2}>0$ such that

$$
\dot{V}_{o} \leq-\frac{k_{q}}{2}\|\tilde{\mathbf{q}}\|_{2}^{2}-c_{2}\|\mathbf{z}\|_{2}^{2}
$$

which implies that there exists $c_{3}>0$ such that

$$
\dot{V}_{o} \leq-c_{3} V_{o}
$$

Therefore, it is concluded that both $\tilde{\mathbf{q}}(t)$ and $\mathbf{z}(t)$ converge to zero exponentially fast. Finally, since

$$
\begin{aligned}
\|\tilde{\boldsymbol{\omega}}(t)\|_{2} & \leq r(t)\|\tilde{\boldsymbol{\omega}}(0)\|_{2} \sqrt{\frac{J_{M}}{J_{m}}} \exp \left\{-\frac{k_{\omega} c_{1}}{J_{M}} t\right\} \\
& \leq \sup _{t \geq 0}\{r(t)\}\|\tilde{\boldsymbol{\omega}}(0)\|_{2} \sqrt{\frac{J_{M}}{J_{m}}} \exp \left\{-\frac{k_{\omega} c_{1}}{J_{M}} t\right\}
\end{aligned}
$$

from Eq. (45), we conclude that

$$
\lim _{t \rightarrow \infty} \tilde{\omega}(t)=\mathbf{0}
$$

for any $\omega(0) \in \mathbb{R}^{3}$ and that the convergence rate is exponential.

Remark 4. As $\tilde{\mathbf{q}}$ converges to zero exponentially fast, so does $\boldsymbol{\beta}$ by Eq. (38). Therefore, we can also conclude that

$$
\lim _{t \rightarrow \infty} C(\mathbf{q}(t)) \bar{\omega}(t)=\omega(t)
$$

with an exponential convergence rate. Then we can interpret $\overline{\boldsymbol{\omega}}$ as the estimate for the angular velocity expressed in the inertial frame $\mathcal{F}_{I}$. 


\section{Full State Feedback Tracking Control}

It is known that PD controllers stabilize the attitude tracking error when all states are available for the controller implementation. Let $\mathbf{q}_{r}(t) \in \mathbb{Q}$ be the quaternion representing the reference frame $\mathcal{F}_{R}$ with respect to the inertial frame $\mathcal{F}_{I}$, and let $\omega_{r}(t) \in \mathbb{R}^{3}$ be the reference angular velocity in the reference frame. Define the quaternion tracking error $\mathbf{e}_{q}=\left(e_{0}, \mathbf{e}_{v}\right) \in \mathbb{Q}$ such that

$$
C\left(\mathbf{e}_{q}\right)=C(\mathbf{q}) C^{T}\left(\mathbf{q}_{r}\right)
$$

and the rate error as

$$
\mathbf{e}_{\omega}=\omega-C\left(\mathbf{e}_{q}\right) \omega_{r}
$$

If the control torque is given by

$$
\mathbf{u}=-k_{p} \mathbf{e}_{v}-k_{v} \mathbf{e}_{\omega}+J C\left(\mathbf{e}_{q}\right) \dot{\omega}_{r}+S\left(\omega_{r}^{B}\right) J \omega_{r}^{B},
$$

where $k_{p}, k_{v}>0$ are the control gains and $\omega_{r}^{B}=C\left(\mathbf{e}_{q}\right) \omega_{r}$ is the reference angular velocity in the body frame, then the tracking error dynamics are obtained as

$$
\begin{aligned}
\dot{\mathbf{e}}_{q} & =\frac{1}{2} E\left(\mathbf{e}_{q}\right) \mathbf{e}_{\omega} \\
J \dot{\mathbf{e}}_{\omega} & =-S\left(\mathbf{e}_{\omega}\right)\left(J \mathbf{e}_{\omega}-\bar{J} \omega_{r}^{B}\right)-k_{p} \mathbf{e}_{v}-k_{v} \mathbf{e}_{\omega} .
\end{aligned}
$$

To prove $\left(\mathbf{e}_{v}, \mathbf{e}_{\omega}\right) \rightarrow(\mathbf{0}, \mathbf{0})$ as $t \rightarrow \infty$, consider the partially strictified Lyapunov function candidate taken from [22]

$$
V_{c}=\zeta_{2} k_{p}\left[\mathbf{e}_{v}^{T} \mathbf{e}_{v}+\left(e_{0}-1\right)^{2}\right]+\frac{\zeta_{2}}{2} \mathbf{e}_{\omega}^{T} J \mathbf{e}_{\omega}+\mathbf{e}_{v}^{T} J \mathbf{e}_{\omega},
$$

where $\zeta_{2}>\frac{k_{v}}{4 k_{p}}+\frac{J_{M}}{2 k_{v}}$. Since

$$
V_{c} \geq \zeta_{2} k_{p}\left(e_{0}-1\right)^{2}+\left[\begin{array}{c}
\mathbf{e}_{v} \\
\mathbf{e}_{\omega}
\end{array}\right]^{T}\left(Q_{3} \otimes J\right)\left[\begin{array}{c}
\mathbf{e}_{v} \\
\mathbf{e}_{\omega}
\end{array}\right]
$$


and

$$
\dot{V}_{c} \leq-\left[\begin{array}{c}
\mathbf{e}_{v} \\
\mathbf{e}_{\omega}
\end{array}\right]^{T}\left(Q_{4} \otimes I_{3}\right)\left[\begin{array}{c}
\mathbf{e}_{v} \\
\mathbf{e}_{\omega}
\end{array}\right],
$$

where the positive definite matrices $Q_{3}$ and $Q_{4}$ are defined by

$$
Q_{3}=\left[\begin{array}{cc}
\frac{\zeta_{2} k_{p}}{J_{M}} & \frac{1}{2} \\
\frac{1}{2} & \frac{\zeta_{2}}{2}
\end{array}\right]
$$

and

$$
Q_{4}=\left[\begin{array}{cc}
k_{p} & \frac{k_{v}}{2} \\
\frac{k_{v}}{2} & \zeta_{2} k_{v}-\frac{J_{M}}{2}
\end{array}\right],
$$

we can conclude, by invoking Barbalat's lemma, that

$$
\lim _{t \rightarrow \infty}\left(\mathbf{e}_{v}(t), \mathbf{e}_{\omega}(t)\right)=(\mathbf{0}, \mathbf{0})
$$

\section{Separation Property}

As the angular velocity measurements are not available, the PD control is modified as

$$
\mathbf{u}=-k_{p} \mathbf{e}_{v}-k_{v} \hat{\mathbf{e}}_{\omega}+J C\left(\mathbf{e}_{q}\right) \dot{\omega}_{r}+S\left(\omega_{r}^{B}\right) J \omega_{r}^{B},
$$

where

$$
\hat{\mathbf{e}}_{\omega}=\hat{\omega}-C\left(\mathbf{e}_{q}\right) \omega_{r}
$$

Consequently, the rate error dynamics are changed to

$$
J \dot{\mathbf{e}}_{\omega}=-S\left(\mathbf{e}_{\omega}\right)\left(J \mathbf{e}_{\omega}-\bar{J} \omega_{r}^{B}\right)-k_{p} \mathbf{e}_{v}-k_{v} \mathbf{e}_{\omega}-k_{v} \tilde{\boldsymbol{\omega}},
$$


which leads to

$$
\dot{V}_{c} \leq-\left[\begin{array}{c}
\mathbf{e}_{v} \\
\mathbf{e}_{\omega}
\end{array}\right]^{T}\left(Q_{4} \otimes I_{3}\right)\left[\begin{array}{c}
\mathbf{e}_{v} \\
\mathbf{e}_{\omega}
\end{array}\right]-k_{v} \mathbf{e}_{v}^{T} \tilde{\boldsymbol{\omega}}-\zeta_{2} k_{v} \mathbf{e}_{\omega}^{T} \tilde{\boldsymbol{\omega}}
$$

To dominate the cross terms in the right hand side of Eq. (73), consider the Lyapunov function candidate

$$
V=V_{c}+\zeta_{3} V_{z}
$$

where $\zeta_{3}$ is a positive constant to be determined. From Eqs. (43) and (73), the upper bound of its time derivative along the closed-loop trajectories is obtained as

$$
\dot{V} \leq-\left[\begin{array}{c}
\mathbf{e}_{v} \\
\mathbf{e}_{\omega} \\
\mathbf{z}
\end{array}\right]^{T}\left(Q_{5} \otimes I_{3}\right)\left[\begin{array}{c}
\mathbf{e}_{v} \\
\mathbf{e}_{\omega} \\
\mathbf{z}
\end{array}\right]
$$

where the matrix $Q_{5}(t) \in \mathbb{R}^{3 \times 3}$ is given by

$$
Q_{5}(t)=\left[\begin{array}{cc}
Q_{4} & R(t) \\
R^{T}(t) & \zeta_{3} c_{1}
\end{array}\right]
$$

with

$$
R(t)=\frac{k_{v} r(t)}{2}\left[\begin{array}{l}
1 \\
\zeta_{2}
\end{array}\right]
$$

Since $Q_{4}$ is positive definite, there exists $c_{4}>0$ such that $Q_{4} \geq c_{4} I_{2}$, discussed earlier. Let $T(t) \in \mathbb{R}^{2 \times 2}$ be the Schur complement of $Q_{4}$ in $Q_{5}(t)$ defined by

$$
T(t)=Q_{4}-\frac{1}{\zeta_{3} c_{1}} R(t) R^{T}(t)
$$

Then the choice

$$
\zeta_{3}>\sup _{t \geq 0}\left\{r^{2}(t)\right\} \frac{k_{v}^{2}\left(1+\zeta_{2}^{2}\right)}{4 c_{1} c_{4}}
$$


makes $T(t)$, and thus, $Q_{5}(t)$ positive definite for all $t \in[0, \infty)$. Applying Barbalat's lemma, we finally have

$$
\lim _{t \rightarrow \infty}\left(\mathbf{e}_{v}(t), \mathbf{e}_{\omega}(t), \mathbf{z}(t)\right)=(\mathbf{0}, \mathbf{0}, \mathbf{0})
$$

\section{Numerical Simulations}

In this section, numerical simulation results are provided to demonstrate the performance of the proposed observer and observer-based PD type tracking controller. The inertia matrix of the rigid spacecraft is taken from [21].

$$
J=\left[\begin{array}{ccc}
10 & 1.2 & 0.5 \\
1.2 & 19 & 1.5 \\
0.5 & 1.5 & 25
\end{array}\right] \mathrm{kg} \mathrm{m}^{2}
$$

The initial conditions are set to

$$
\mathbf{q}_{v}(0)=\left[\begin{array}{c}
-0.1 \\
0.1 \\
-0.1
\end{array}\right], \quad \omega(0)=\left[\begin{array}{c}
0.005 \\
0.006 \\
0.004
\end{array}\right] \mathrm{rad} / \mathrm{s}
$$

with $q_{0}=\sqrt{1-\left\|\mathbf{q}_{v}(0)\right\|_{2}^{2}}$. The reference quaternion is set to $\mathbf{q}_{r}(0)=(0.9487,0.1826,0.1826,0.1826)$ initially and its dynamics are assumed to be driven by the reference angular velocity profile $\omega_{r}(t)=$ $\left(\omega_{r}(t), \omega_{r}(t), \omega_{r}(t)\right) \mathrm{rad} / \mathrm{s}$, where

$$
\omega_{r}(t)=0.3 \cos (t)\left(1-e^{-0.01 t^{2}}\right)+(0.08 \pi+0.006 \sin (t)) t e^{-0.01 t^{2}} .
$$

To show the tracking performance, we set the control gains to $k_{p}=1.5$ and $k_{v}=5$. For the observer, we use

$$
k_{1}=\frac{1}{4}, \quad k_{2}=\frac{\left(J_{M}+2 \sqrt{J_{m} J_{\Delta}}\right)^{2}}{4 J_{m}^{2}\left(1-2 k_{1}\right)}
$$

The gains $k_{q}$ and $k_{\omega}$ are closely related to the convergence rate of the observer state errors. In the simulation, they are selected such that the convergence rates for the quaternion and angular velocity errors are approximately the same. For comparison, two sets of these gains are chosen: 
$\left(k_{q}, k_{\omega}\right)=(0.3,0.3)$ and $(15,15)$. The initial guess for the angular velocity is selected as $\hat{\omega}(0)=$ $(-0.3,0.1,-0.2) \mathrm{rad} / \mathrm{s}$.

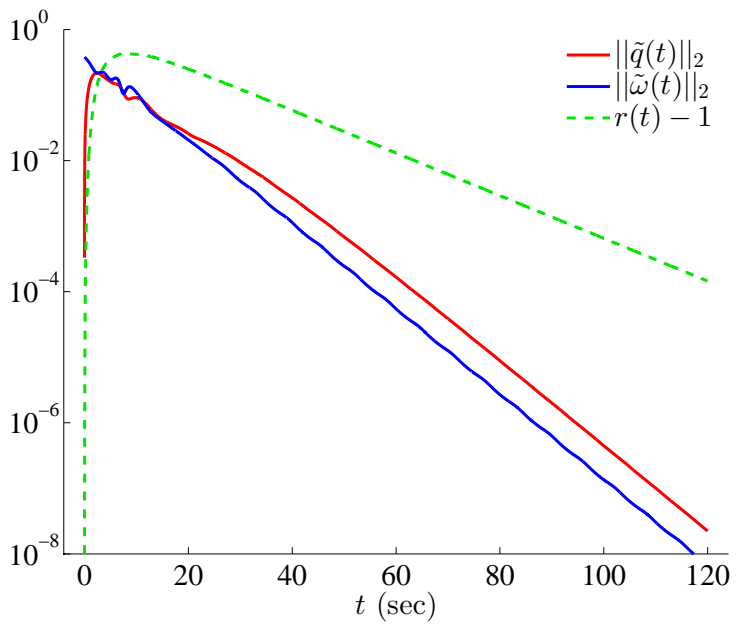

a) $k_{q}=0.3, k_{\omega}=0.3$

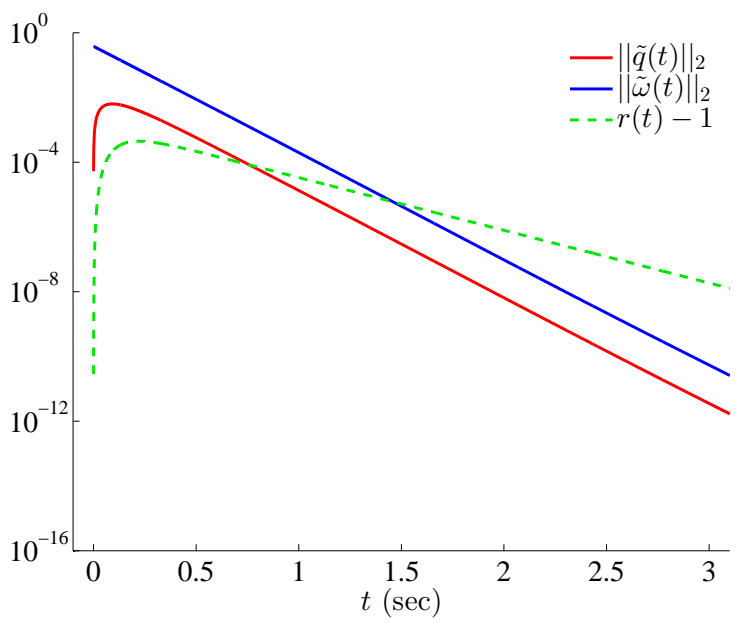

b) $k_{q}=15, k_{\omega}=15$

Figure 1. Time histories of observer errors

The performance of the proposed observer is shown in Fig. 1. The high gains ensure a fast convergence rate as expected. Given fixed gains $k_{q}$ and $k_{\omega}$, the convergence rate of the scaling factor is further restricted by the other observer gains. So the scaling factor converges faster to unity if $k_{1}$ and $k_{2}$ are close to their constraint boundaries described in Eqs. (41) and (42).

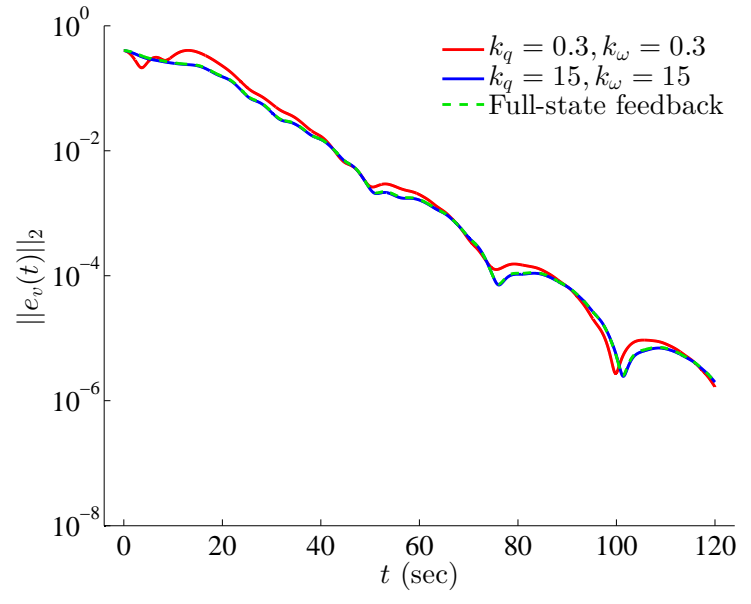

a) Quaternion errors

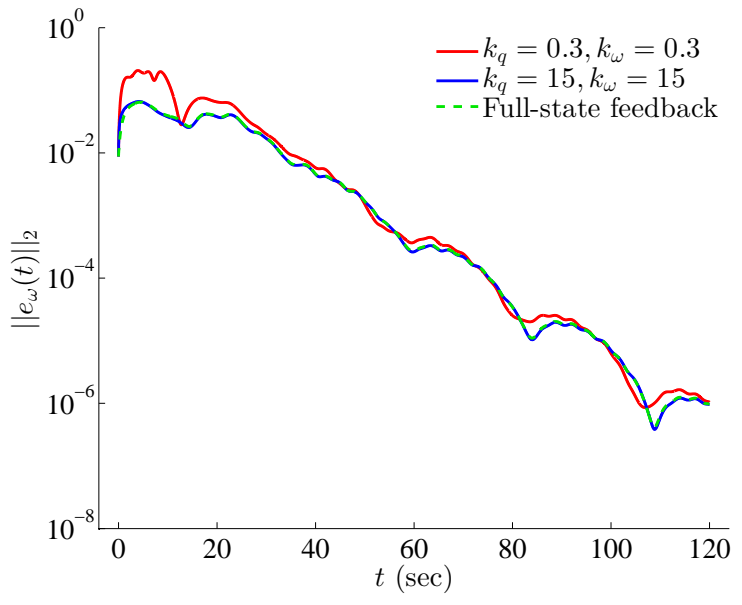

b) Angular velocity errors

Figure 2. Time histories of tracking errors

The tracking performance of the observer-based PD controller is demonstrated and compared with the full-state feedback control in Fig. 2. When the small gain set is selected for the observer, 
the tracking error norms are deviated from those of the full-state feedback control, but the convergence rate is not very different because the convergence rate of the observer errors is still faster than that of the tracking errors in the ideal PD control. When the observer gains are increased to the values in the second set, the trajectories are almost identical to the full-state feedback control. Figure 3 also shows the same tendency in the control effort. For the high gain observer, the trajectory of the control torque norm catches up the full-state feedback control within a second.

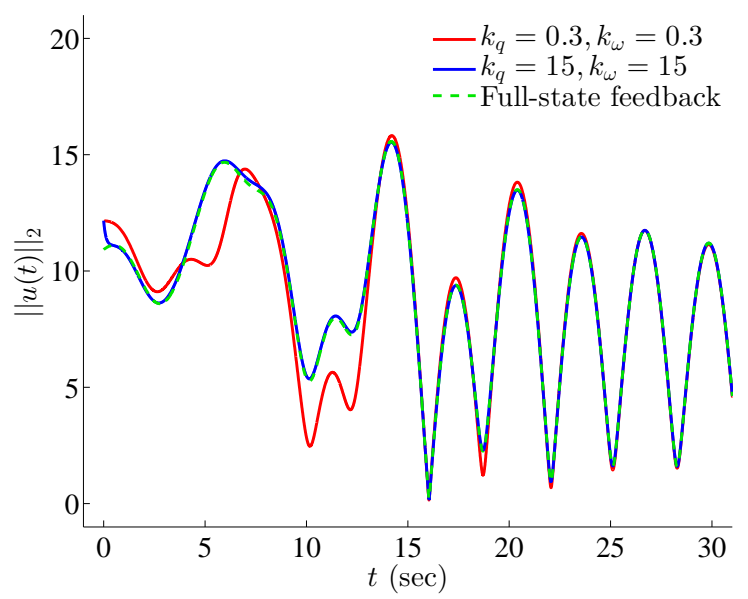

Figure 3. Time histories of control torques

\section{Conclusion}

In this note, a novel observer-based attitude tracking controller is proposed in the absence of rate measurements. Based on the I\&I design method in conjunction with dynamic scaling, an angular velocity observer employing the attitude quaternion measurements is designed. It is shown that the angular velocity estimate globally converges to the true value exponentially fast independent of the choice of control torque. By defining the rate estimate using a direction cosine matrix, the observer structure is far simplified and requires less state variables compared to the existing I\&I design. A separation property is then established for the PD tracking controller while asymptotic convergence of the tracking errors is shown. To demonstrate the observer performance, numerical simulations are conducted. Tracking results of two observers with relatively high and low gains are provided and compared with the ideal case where true angular velocity is available for feedback control. 


\section{References}

[1] Wen, J.-Y. and Kreutz-Delgado, K., "The attitude control problem," Automatic Control, IEEE Transactions on, Vol. 36, No. 10, 1991, pp. 1148-1162.

[2] Wie, B., Space Vehicle Dynamics and Control, AIAA Education Series, AIAA, Reston VA, 2nd ed., 2008, ISBN: 978-1-56347-953-3.

[3] Lizarralde, F. and Wen, J. T., "Attitude control without angular velocity measurement: A passivity approach," Automatic Control, IEEE Transactions on, Vol. 41, No. 3, 1996, pp. 468-472.

[4] Tsiotras, P., "Further passivity results for the attitude control problem," IEEE Transactions on Automatic Control, Vol. 43, No. 11, 1998, pp. 1597-1600.

[5] Akella, M. R., "Rigid body attitude tracking without angular velocity feedback," Systems \& Control Letters, Vol. 42, No. 4, 2001, pp. 321-326.

[6] Costic, B., Dawson, D., De Queiroz, M., and Kapila, V., "Quaternion-based adaptive attitude tracking controller without velocity measurements," Journal of Guidance, Control, and Dynamics, Vol. 24, No. 6, 2001, pp. 1214-1222.

[7] Akella, M. R., Valdivia, A., and Kotamraju, G. R., "Velocity-free attitude controllers subject to actuator magnitude and rate saturations," Journal of Guidance, Control, and Dynamics, Vol. 28, No. 4, 2005, pp. 659-666.

[8] Cai, W., Liao, X., and Song, D. Y., "Indirect robust adaptive fault-tolerant control for attitude tracking of spacecraft," Journal of Guidance, Control, and Dynamics, Vol. 31, No. 5, 2008, pp. 1456-1463.

[9] Seo, D. and Akella, M. R., "High-performance spacecraft adaptive attitude-tracking control through attracting-manifold design," Journal of Guidance, Control, and Dynamics, Vol. 31, No. 4, 2008, pp. 884-891.

[10] Tayebi, A., Roberts, A., and Benallegue, A., "Inertial vector measurements based velocity-free attitude stabilization," Automatic Control, IEEE Transactions on, Vol. 58, No. 11, 2013, pp. 2893-2898.

[11] Thakur, D. and Akella, M. R., "Gyro-free rigid-body attitude stabilization using only vector measurements," Journal of Guidance, Control, and Dynamics, Vol. 38, No. 4, 2014, pp. 811-818.

[12] Salcudean, S., "A globally convergent angular velocity observer for rigid body motion," Automatic Control, IEEE Transactions on, Vol. 36, No. 12, 1991, pp. 1493-1497.

[13] Nicosia, S. and Tomei, E., "Nonlinear observer and output feedback attitude control of spacecraft," Aerospace and Electronic Systems, IEEE Transactions on, Vol. 28, No. 4, 1992, pp. 970-977.

[14] Hu, J. and Zhang, H., "Bounded output feedback of rigid-body attitude via angular velocity observers," Journal of Guidance, Control, and Dynamics, Vol. 36, No. 4, 2013, pp. 1240-1248.

[15] Karagiannis, D., Sassano, M., and Astolfi, A., "Dynamic scaling and observer design with application to adaptive control," Automatica, Vol. 45, No. 12, 2009, pp. 2883-2889.

[16] Astolfi, A. and Ortega, R., "Immersion and invariance: a new tool for stabilization and adaptive control of nonlinear systems," Automatic Control, IEEE Transactions on, Vol. 48, No. 4, 2003, pp. 590-606.

[17] Astolfi, A., Karagiannis, D., and Ortega, R., Nonlinear and adaptive control with applications, Springer Science \& Business Media, 2007. 
[18] Astolfi, A., Ortega, R., and Venkatraman, A., "A globally exponentially convergent immersion and invariance speed observer for n degrees of freedom mechanical systems," Decision and Control, 2009 held jointly with the 2009 28th Chinese Control Conference. CDC/CCC 2009. Proceedings of the 48th IEEE Conference on, IEEE, 2009, pp. 6508-6513.

[19] Astolfi, A., Ortega, R., and Venkatraman, A., "A globally exponentially convergent immersion and invariance speed observer for mechanical systems with non-holonomic constraints," Automatica, Vol. 46, No. 1, 2010, pp. 182-189.

[20] Chunodkar, A. A. and Akella, M. R., "Switching angular velocity observer for rigid-body attitude stabilization and tracking control," Journal of Guidance, Control, and Dynamics, Vol. 37, No. 3, 2014, pp. 869-878.

[21] Akella, M. R., Thakur, D., and Mazenc, F., "Partial Lyapunov Strictification: Smooth Angular Velocity Observers for Attitude Tracking Control," Journal of Guidance, Control, and Dynamics, Vol. 38, No. 3, 2015, pp. 442-451.

[22] Mazenc, F. and Akella, M. R., "Quaternion-based stabilization of attitude dynamics subject to pointwise delay in the input," American Control Conference (ACC), 2014, IEEE, 2014, pp. 4877-4882. 